\title{
Importance of the carboxyl terminus for folding and trafficking of the serotonin transporter
}

\author{
Ali El-Kasaby* and Oliver Kudlacek
}

\author{
Address: Institute of Pharmacology, Center for Biomolecular Medicine and Pharmacology, Medical University of Vienna, 1090 Vienna, Austria \\ Email: Ali El-Kasaby* - ali.el-kasaby@meduniwien.ac.at \\ * Corresponding author
}

\begin{abstract}
from I5th Scientific Symposium of the Austrian Pharmacological Society (APHAR) Joint meeting with the Hungarian Society of Experimental and Clinical Pharmacology (MFT) and the Slovenian Pharmacological Society (SDF)

Graz, Austria. 19-21 November 2009

Published: 12 November 2009

BMC Pharmacology 2009, 9(Suppl 2):A27 doi:10.1186/147I-2210-9-S2-A27
\end{abstract}

This abstract is available from: http://www.biomedcentral.com//47I-22I0/9/S2/A27

(c) 2009 El-Kasaby and Kudlacek; licensee BioMed Central Ltd.

\section{Background}

The human serotonin transporter is the plasma membrane $\mathrm{Na}^{+} / \mathrm{Cl}^{-}$-dependent transporter responsible for uptake of serotonin from the synaptic cleft.

\section{Methods}

We studied the importance of the carboxyl terminus in folding and trafficking of the serotonin transporter by generation of SERT mutants by site-directed mutagenesis (alanine scanning mutagenesis), transient expression in HEK293 cells, localization by epifluorescence microscopy and confocal laser scanning microscopy, biochemical characterization (binding studies, uptake studies) and test for possible pharmacochaperoning effect of SERT ligands.

\section{Results}

Our data show that the mutation in P601G602-AA, R607I608-AA and RII-AAA (Sec24 binding site) causes intracellular retention and abolishes uptake and binding. We could rescue the mutant (RI-AA and RII-AAA) by ibogaine $(100 \mathrm{mM})$ and DMSO (2\%) but not with 5-HT $(100 \mathrm{mM})$, imipramine $(10 \mathrm{mM})$ or low temperature $\left(31^{\circ} \mathrm{C}\right)$. However, the mutant PG-AA could not be rescued by any of these compounds. We studied the effect of the mutations on mis-folding by expression of our mutants in a bacterial system [1] but we could not use this protocol for SERT, so we turned to co-immunopreciptation of SERT (wild-type and mutant) with calnexin antibody as has been described by Duvernay et al. [2], and we could immunopreciptate calnexin (preliminary data).

\section{Acknowledgements}

This work was supported by SFB35-10 and a stipend from Egyptian Ministry of Higher Education and State for Scientific Research.

\section{References}

I. Waldo GS, Standish BM, Berendzen J, Terwilliger TC: Rapid protein-folding assay using green fluorescent protein. Nat Biotechnol 1999, 17:69|-695.

2. Duvernay MT, Dong C, Zhang X, Zhou F, Nichols CD, Wu G: Anterograde trafficking of $G$ protein-coupled receptors: function of the C-terminal $F(X)_{6} L L$ motif in export from the endoplasmic reticulum. Mol Pharmacol 2009, 75:75I-76I. 